\title{
Numerical investigation of the impact of mesoscale air-sea interaction on atmosphere
}

\author{
Huang liwen ${ }^{1.3}$ \\ ${ }^{1}$ Wuhan University of Technology \\ Wuhan, China \\ ${ }^{3}$ Institute of Atmospheric Physics, CAS \\ Beijing, China \\ lwhuang@whut.edu.cn
}

\author{
Ge yijun ${ }^{1,2}$ \\ ${ }^{2}$ Naval University of Engineering \\ Wuhan, China \\ geyijun1983@163.com
}

\begin{abstract}
Using the mesoscale coupled air-sea model, Typhoon Winnie (1997) is numerical simulated to investigated the impact of air-sea interaction on atmosphere. Compared with uncoupled model, the simulated results of typhoon intensity from coupled model become more realistic, with decreases in central pressure error of $2.3 \mathrm{hPa}$ at $48 \mathrm{hr}$ and $2.8 \mathrm{hPa}$ at $72 \mathrm{hr}$. The air-sea interaction noticeably weakened the warm core of the typhoon. In the typhoon center, the air-sea interaction resulte in a temperature cooling of $3^{\circ} \mathrm{C}$ in the lower layer of the atmosphere and $2^{\circ} \mathrm{C}$ in the 500 to $600 \mathrm{hPa}$ layers. In the mean time, the relative humidity can be decreased by 10 to $30 \%$. Furthermore, the air-sea interaction leads to change of the distribution of air temperature, wind field and precipitation near the sea surface.
\end{abstract}

Index Terms-Typhoon, air-sea interaction, mesoscale coupled model

\section{INTRODUCTION}

Air-sea interaction is one of the main focuses on the research of Typhoon activity. Developing a mesoscale coupling model is a reasonable approach to investigate these complicated processes. There have great progress on the research of regional air-sea coupled models[1]. With the developed mesoscale coupling model, many interacting processes can be studied. Bender and Ginis [2] examined the effects of tropical cyclone-ocean interaction on the intensity of observed storms using a coupled hurricane-ocean model. Emanuel et al. [3] explored the positive and negative effects of various environmental factors on tropical cyclone intensity.

So far, minimal work has been carried out on the Yellow and East China Seas (YECS). Therefore, the primary objective of this paper is to analyze the mesoscale two-way interaction between atmosphere and ocean during the passage of a summer typhoon over this area using a developed Mesocale Coupled model (MCM [4]). The MCM is based on two independent models. The atmospheric model is the Mesoscale Model (MM5 [5]) developed by Pennsylvania State University/the National Center of Atmospheric Research. This model is a limited-area, non-hydrostatic, terrain following sigma-coordinate model designed to simulate or predict mesoscale atmospheric circulation. The oceanic model is an updated Estuary, Coast and Ocean Model (ECOM-si [6]). It is a three-dimensional, fully nonlinear, primitive equation ocean model. To couple MM5 with ECOM-si, we use a concurrent process communication technique to establish the linkage between the two models, by which the momentum flux (wind stress) and heat fluxes (including latent heat flux, sensible heat flux, short and long wave radiation fluxes) are passed from the atmospheric model to the oceanic model, or vice versa for SST. The research in this study is focused on the impact of air-sea interaction on atmosphere.

\section{CASE}

We selected the evolution of Typhoon Winnie, which occurred between 0000 UTC 8 and 0000 UTC 20 August 1997 as a case study. Typhoon Winnie was a typical landing typhoon that lasted for a long time with a strong intensity. It formed at about 0000 UTC 8 August 1997, then moved northwestward and developed rapidly. The center of Typhoon Winnie entered into the oceanic model domain at 1200 UTC 16 August 1997 and slowly moved northwestward with a constant center pressure of $960 \mathrm{hPa}$ (Fig.1). At about 1400 UTC 18 August, it landed at Wenling, Zhejiang Province, and then moved northward across eastern China and evolved into an extratropical cyclone.

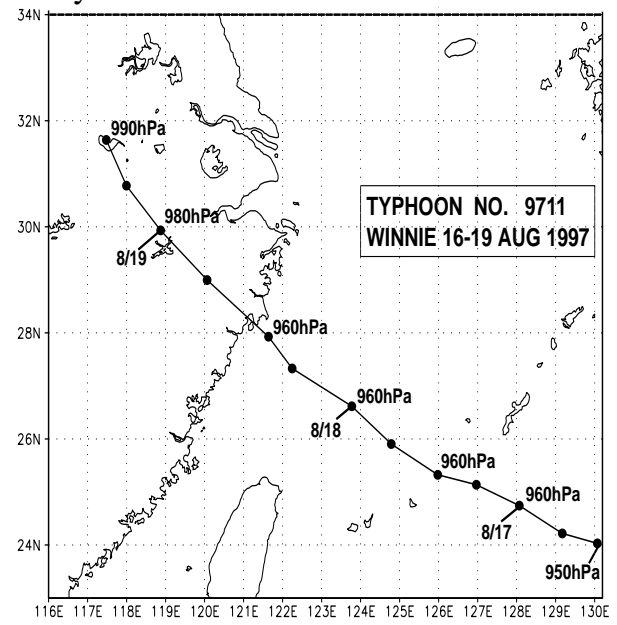

Fig. 1. Track of Typhoon Winnie(1997) 


\section{EXPERIMENT DESIGN}

In this paper, the center of the atmospheric model domain is $33^{\circ} \mathrm{N}, 125^{\circ} \mathrm{E}$, and the grid system is constructed with $187 \times 141$ horizontal grid points at a medium resolution of $37.5 \mathrm{~km}$. The oceanic model domain is positioned from $23^{\circ} \mathrm{N}$ to $41^{\circ} \mathrm{N}$ latitude and $116^{\circ} \mathrm{E}$ to $131^{\circ} \mathrm{E}$ longitude, which covers the entire Yellow and East China Seas. A rectangular grid is constructed with $164 \times 202$ horizontal grid points at a high resolution of 10 $\mathrm{km}$ for the ocean model. In the vertical direction, there are 15 sigma levels for MM5 while 11 sigma levels for ECOM-si from the water surface to the bottom. The time step for MM5 and ECOM-si is $120 \mathrm{~s}$ and $480 \mathrm{~s}$ respectively. Both the atmospheric and oceanic models are initialized at 1200 UTC 16 August 1997 and are simultaneously run forward in time for 72 hr. The first experiment, denoted EXP1, is the single atmospheric model running with a fixed SST field. The second, denoted EXP2, is the mesoscale coupling model running for 72 hr.

\section{EXPERIMENTAL RESULTS}

\section{A. Track and intensity}

The location and sea surface pressure of typhoon center from observation and numerical simulations are compared in Fig2. The typhoon tracks of both experiments are similar to the observation at the first $24 \mathrm{hr}$ (Fig.2a). During $24 \mathrm{hr}$ to $48 \mathrm{hr}$, the typhoon moves northwestward in both simulations, which is consistent with the moving direction of Typhoon Winnie from observation, but the location of the center is on the north side of the observation. After 1200 UTC 18 August, the tracks in experiments suddenly turn right, then move almost northward along the coastline. The landing process is not simulated in EXP1. It is improved in EXP2 after taking the air-sea interaction into consideration, while the landing place is away from the observation. For the minimum Sea Level Pressure of typhoon center (Fig.2b), which donates the intensity of typhoon, both simulation results show the weakening process of typhoon Winnie. EXP2 performs better than EXP1 during $36 \mathrm{hr}$ to $72 \mathrm{hr}$. Compared to EXP1, The center pressure of EXP2 is higher by $2.3 \mathrm{hPa}$ for 1200 UTC 18 August and $2.8 \mathrm{hPa}$ for 1200 UTC 19 August. It should be noted that there are almost no difference in track and intensity between EXP1 and EXP2 in the first $36 \mathrm{hr}$ (before 0000 UTC 18 August). On the one hand, this may be caused by the fact that the typhoon center is out of the domain of ocean model at the beginning. On the other hand, it might indicate that the mesoscale air-sea interaction is an accumulated process, so its effect is expressed during the later part of the simulation period.

\section{B. Atmosphere vertical structure}

The air-sea interaction have impact on the atmosphere vertical structure during the period when typhoon Winnie passed. Fig. 3 presents the vertical distribution of the temperature and relative humidity in EXP2 at $48 \mathrm{hr}$ along the section of typhoon center, as well as the difference of above variables between EXP2 and EXP1. The air-sea interaction noticeably weakened the warm core of the typhoon. In the typhoon center, the air-sea interaction resulted in a temperature cooling of $3^{\circ} \mathrm{C}$ in the lower layer of the atmosphere and $2{ }^{\circ} \mathrm{C}$ in the 500 to $600 \mathrm{hPa}$ layers. This influence can even be extended to the high layer of $400 \mathrm{hPa}$, and the relative humidity can be decreased by 10 to $30 \%$. The ascending movement is restrained while the descending movement is strengthened. This could be the main reason why the typhoon weakens more rapidly when the air-sea interaction is considered.

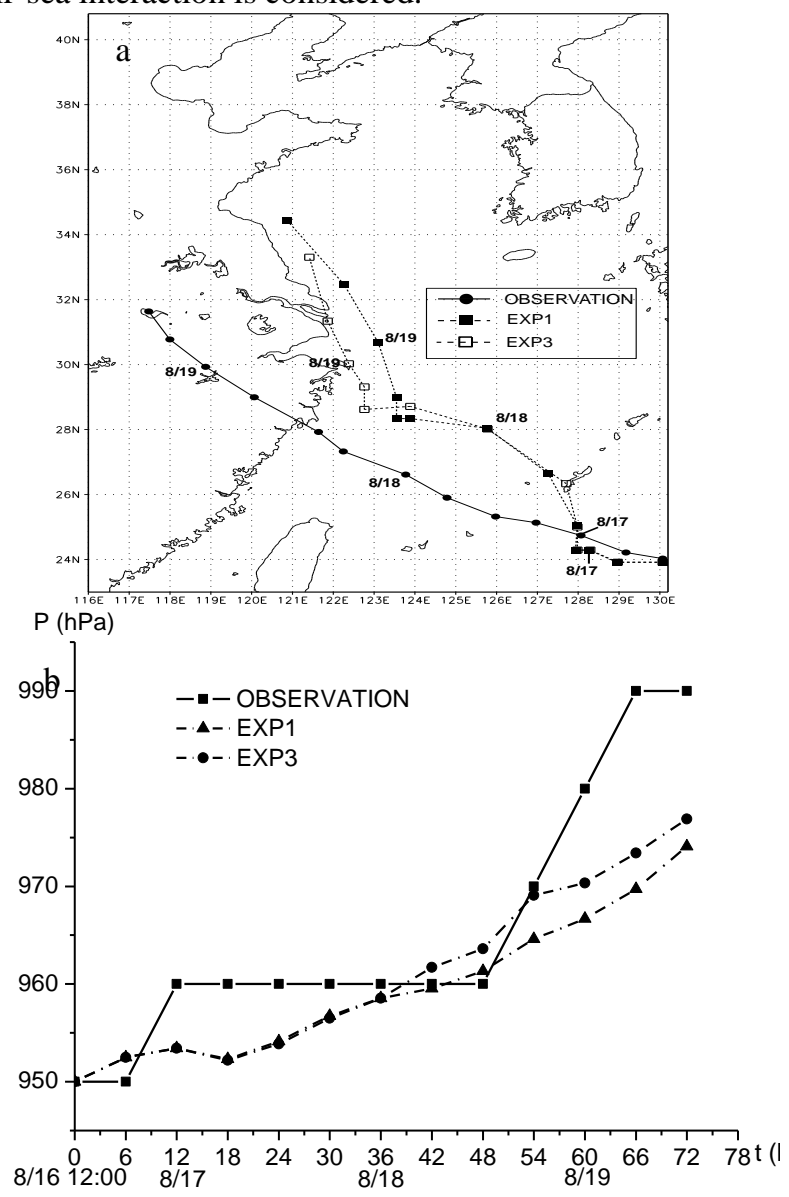

Fig. 2. Comparison of the typhoon track(a) and minimum Sea Level Pressure(b) among observation、 EXP1 and EXP3. 


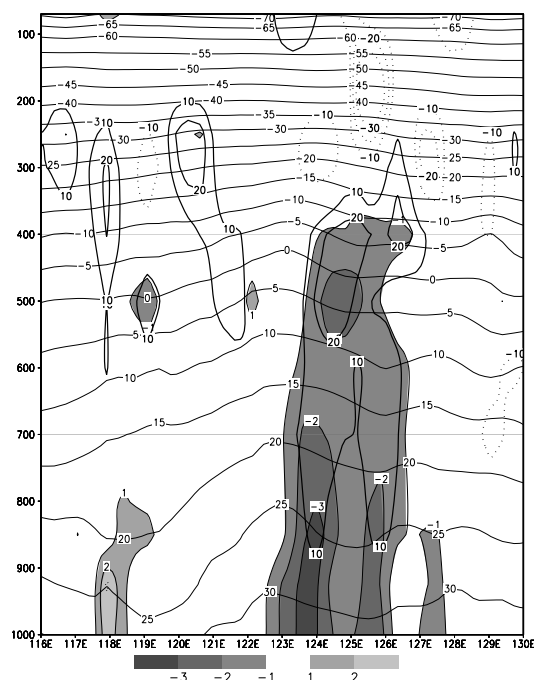

Fig. 3. Vertical cross-section passing through the center of Typhoon Winnie at 1200 UTC 18 August. The thick contour with interval of $10^{\circ} \mathrm{C}$ represent the temperature, the darker shading indicate temperature difference, the thin solid line is the relative humidity while the dot line denotes the difference of relative humidity;

\section{Variations near the sea surface}

The distribution of air temperature and wind field near the sea surface also change noticeably because of the air-sea interaction. Fig.4a gives the difference of wind velocity near the sea surface between EXP2 and EXP1 at 0000 UTC 18 August when air-sea interaction begins to take effect as discussed above. It shows that the wind velocity increased by $4 \mathrm{~m} / \mathrm{s}$ near the typhoon center, while on the south side of its center the wind velocity decreased more than $5 \mathrm{~m} / \mathrm{s}$ (shading area). The air temperature decreased more than $3^{\circ} \mathrm{C}$ on left side of the typhoon track(Fig.4b).

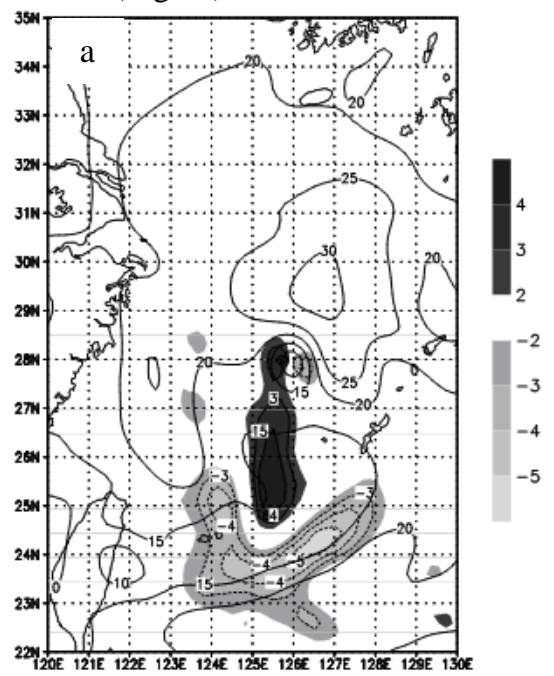

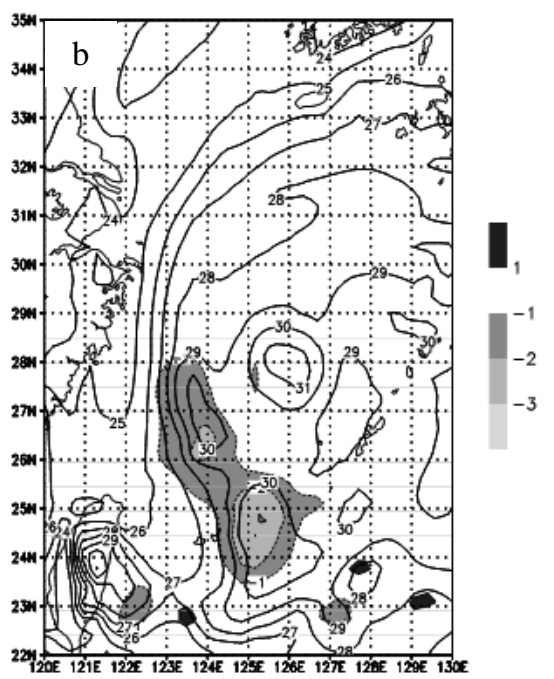

Fig. 4. (a)wind speed in EXP2 (contours every $5 \mathrm{~m} / \mathrm{s}$ ) and difference of wind velocity between EXP2 and EXP1 (shading) at 0000 UTC 18 August; (b)same with (a), but for temperature. contour interval with $1^{\circ} \mathrm{C}$.

\section{Precipitation}

Furthermore, the numerical simulated results are influenced by the air-sea interaction in term of the time, location and intensity of precipitation. Fig.5a presents total precipitation at 1200 UTC 18 August. the center position of rainfall is consistent to that from NCEP reanalysis datasets(not show in this paper), especially as for the area with heavy precipitation exceed $150 \mathrm{~mm}$ which located southeastern side of the typhoon. However, there are two more area of rainfall in EXP2, one located in the southeastern of island and the other in the Taiwan Island. The former can hardly validated because of no observation of precipitation over the sea. However, it is observed that over the Taiwan Island the rain mainly begin from 0000 UTC, 17 to 0000 UTC, 18 August, and precipitation area located in the center and northern of Taiwan Island with the value exceed $400 \mathrm{~mm}$. The distribution and intensity of rainfall in EXP3 during the same time is indicated by Fig. 5b. It can be seen that the total precipitation exceed $400 \mathrm{~mm}$ also, while the center position located near the southern of island. This difference partly results from the error of simulated typhoon center. There is another rainfall area located near the Changjiang Estuary and HangZhou bay. 

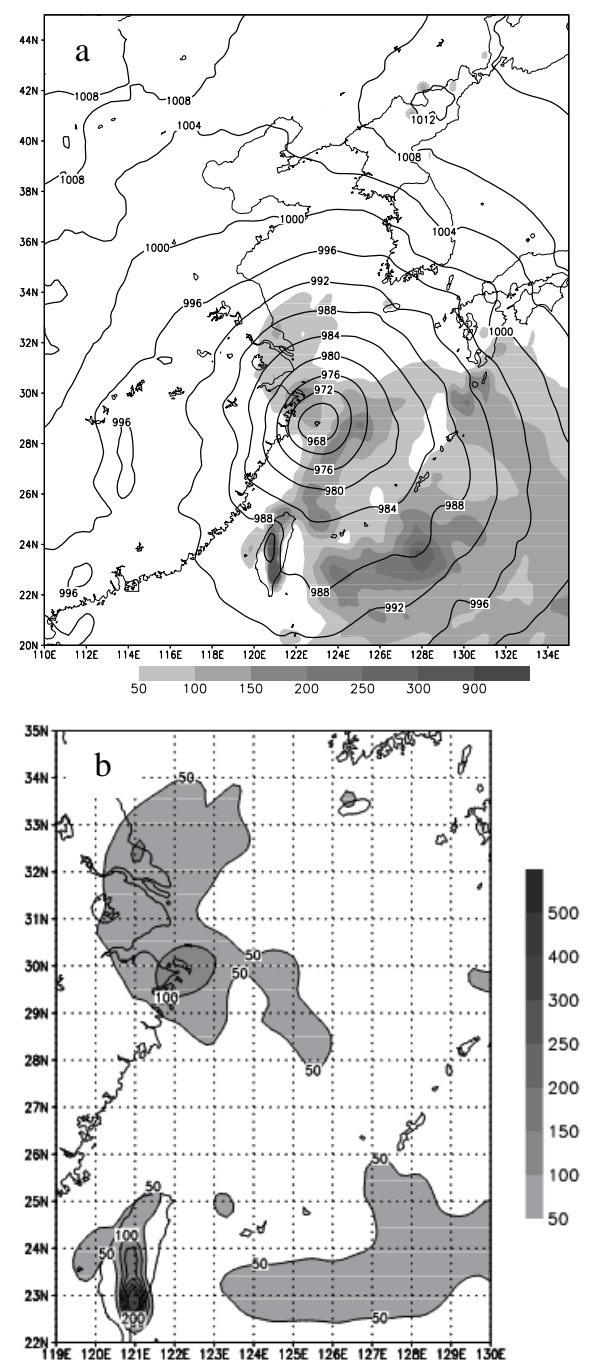

Fig. 5. (a)Sea surface pressure(contour every $4 \mathrm{hPa}$ ) and total precipitation (shading, mm) in EXP2 at 1200 UTC 18 August;(b)24-h precipitation(mm) from 1200 UTC 17 to 1200 UTC 18 August

\section{SUMMARY}

Using the mesoscale coupled air-sea model LASG-MCM, Typhoon Winnie(1997) is numerical simulated to investigated the impact of air-sea interaction on atmosphere. Compared with uncoupled model, the simulated result of typhoon intensity from coupled model becomes more realistic, with decreases in central pressure error of $2.3 \mathrm{hPa}$ at 1200 UTC 18 August and $2.8 \mathrm{hPa}$ at 1200 UTC 19 August. The air-sea interaction noticeably weakened the warm core of the typhoon. In the typhoon center, the air-sea interaction resulted in a temperature cooling of $3^{\circ} \mathrm{C}$ in the lower layer of the atmosphere and $2^{\circ} \mathrm{C}$ in the 500 to $600 \mathrm{hPa}$ layers, In the mean time, the relative humidity can be decreased by 10 to $30 \%$. The air-sea interaction further leads to change of the distribution of air temperature and wind field near the sea surface, as well as the precipitation.

\section{ACKNOWLEDGMENT}

This work was financially supported by the National High Technology Research and Development Programs of China (863 Program, Grant No. 2010AA012300).

\section{REFERENCES}

[1] Peng S Q, Liu D L, Sun Z B, et al. Recent advances in regional air-sea coupled models. Sci China Earth Sci, 2012, 55: 13911405(in Chinese)

[2] Bender, M. A. and Ginis, I.. Real case simulations of hurricaneocean interaction using a high resolution coupled model: Effect on hurricane intensity. Mon. Wea. Rev., 2000, 128(4):917-946.

[3] Emanuel, K., DesAutels, C., Holloway, C., and Korty, R. Environmental control of tropical cyclone intensity. Journal of the Atmosphere Sciences, 2004, 61(7): 843-858

[4] Qi C X, Huang L W, Wu G X, Yu R C. Numerical experiment of a coupled air-sea mesoscale model MM5V3/Ecom-si. Journal of Wuhan University of Technology, 2003,27(4):443-448(in Chinese)

[5] Dudhia, J., D. Gill, Y-R.Guo et al., PSU/NCAR Mesoscale Modeling System Tutorial Class Notes: MM5 Modeling System Version 2,1998.

[6] Hydroqual Inc., A Primer for ECOM-si,1991 